\title{
A Consistent Diffusion-Dissipation Parameterization in the ECHAM Climate Model
}

\author{
ULRIKE BURKHARDT \\ Institut für Physik der Atmosphäre, DLR Oberpfaffenhofen, Wessling, Germany \\ ERICH BECKER \\ Leibniz-Institut für Atmosphärenphysik, Kühlungsborn, Germany
}

(Manuscript received 15 February 2005, in final form 29 June 2005)

\begin{abstract}
The diffusion-dissipation parameterizations usually adopted in GCMs are not physically consistent. Horizontal momentum diffusion, applied in the form of a hyperdiffusion, does not conserve angular momentum and the associated dissipative heating is commonly ignored. Dissipative heating associated with vertical momentum diffusion is often included, but in a way that is inconsistent with the second law of thermodynamics.

New, physically consistent, dissipative heating schemes due to horizontal diffusion (Becker) and vertical diffusion (Becker, and Boville and Bretherton) have been developed and tested. These schemes have now been implemented in 19- and 39-level versions of the ECHAM4 climate model. The new horizontal scheme requires the replacement of the hyperdiffusion with a $\nabla^{2}$ scheme.

Dissipation due to horizontal momentum diffusion is found to have maximum values in the upper troposphere/lower stratosphere in midlatitudes and in the winter hemispheric sponge layer, resulting in a warming of the area around the tropopause and of the polar vortex in Northern Hemispheric winter. Dissipation associated with vertical momentum diffusion is largest in the boundary layer. The change in parameterization acts to strengthen the vertical diffusion and therefore the associated dissipative heating. Dissipation due to vertical momentum diffusion has an indirect effect on the upper-tropospheric/stratospheric temperature field in northern winter, which is to cool and strengthen the northern polar vortex. The warming in the area of the tropopause resulting from the change in both dissipation parameterizations is quite similar in both model versions, whereas the response in the temperature of the northern polar vortex depends on the model version.
\end{abstract}

\section{Introduction}

A key role of momentum diffusion in climate models is to transfer kinetic energy from the resolved into the unresolved scales. In a GCM, diffusion is split into a vertical and a horizontal component. Vertical diffusion parameterizes turbulent, nonconvective processes that are most important in the boundary layer. Horizontal diffusion parameterizes the energy transfer from wave disturbances into the nonresolved scales, preventing an accumulation of energy and enstrophy at the model grid scale (Pedlosky 1987, 246-253). It is therefore at a

Corresponding author address: Ulrike Burkhardt, Institut für Physik der Atmosphäre, DLR Oberpfaffenhofen, 82234 Wessling, Germany.

E-mail: ulrike.burkhardt@dlr.de maximum in the upper troposphere in midlatitudes and in the sponge layer of a model.

The loss of kinetic energy due to diffusion must theoretically be balanced by dissipative heating (equivalent terms are frictional heating or dissipation) in order to conserve energy. Dissipation due to vertical momentum diffusion (hereafter referred to as vertical dissipation) is often included assuming a local equivalence of the energy lost by momentum diffusion and gained through dissipative heating [e.g., DKRZ 1992; Hamilton 1996; Medvedev and Klaasen 2003; see also the European Centre for Medium-Range Weather Forecasts (ECMWF) Integrated Forecast System (IFS) CY28r1 at http://www.ecmwf.int/research/ifsdocs/ CY28r1/Physics, section 3.6]. Dissipative heating due to horizontal momentum diffusion (hereafter referred to as horizontal dissipation) is usually ignored or other- 
wise incorporated analogously to the vertical dissipation (Kiehl et al. 1996).

Horizontal and vertical diffusion and dissipation can be formulated in an energy- and angular-momentumconserving way that guarantees dissipative heating to be positive definite and therefore the parameterized energy transfer to be irreversible in the thermodynamic sense. This constraint is violated in parameterizations of vertical dissipation that assume a local equivalence of energy lost (gained) by momentum diffusion and gained (lost) by dissipative heating. Vertical dissipation must rather be based on the stress tensor used for vertical momentum diffusion (Becker 2003; Boville and Bretherton 2003). This causes a different distribution of the heating within a model column. Horizontal dissipation is only positive definite if horizontal diffusion is not a hyperdiffusion but of second order (Becker 2001). Since the introduction of harmonic diffusion reduces the scale selectivity of the diffusion-damping not only the short but also the larger-scale waves-second-order diffusion is usually not used in coarse-resolution climate models. A way to make the horizontal diffusiondissipation parameterization consistent while retaining a stronger-scale selectivity might be the introduction of a nonlinear $\nabla^{2}$ diffusion (Smagorinsky 1993). Nevertheless, as long as the overall amount of dissipative heating is realistic, we can assess the importance of the dissipation parameterization using a linear harmonic horizontal diffusion.

The amount of kinetic energy lost due to diffusion can be assumed to balance, on the climatological time scale, the amount of kinetic energy generated through conversion of available potential energy and consequently the available potential energy generated through differential heating. The net generation of large-scale available potential energy, and therefore dissipative heating, is estimated from observations to be about $2 \mathrm{~W} \mathrm{~m}^{-2}$ (Oort 1964). According to simpleGCM estimates (Becker 2003), total dissipation amounts to about $2 \mathrm{~W} \mathrm{~m}^{-2}$, with horizontal dissipation making up about a third of the overall dissipation. Neglecting this heating therefore causes an artificial thermal forcing of $\sim 0.6 \mathrm{~W} \mathrm{~m}^{-2}$. This value may be smaller than the inconsistencies in other physical parameterizations such as phase transitions or convection parameterization, but nevertheless is of importance since it is the same order of magnitude as the forcing due to climate change.

In runs with a dry simple GCM, Becker $(2001,2003)$ showed that including the dissipative heating due to momentum diffusion eliminated spurious thermal forcing and angular momentum sources both in life cycle experiments and in climate runs. Including dissipative heating versus neglecting it in climate simulations with the simple GCM resulted in changes in the simulated mean temperature field of about $1.5 \mathrm{~K}$ in the stratospheric NH winter polar vortex and an associated change in the zonal wind pattern. Those results indicated that dissipative heating might be of importance also for the climatology simulated by a sophisticated GCM.

In this paper we shall examine how the simulated climate of the ECHAM4 climate model changes when adopting the new schemes for horizontal and vertical dissipation. The paper sets out to show that the reformulation of vertical dissipation matters and that it should therefore be generally reformulated in GCMs. Horizontal dissipation, on the other hand, is implemented in combination with the harmonic horizontal diffusion. Since low-order diffusion, such as harmonic diffusion, have detrimental effects on the simulated large-scale climate in coarse-resolution models (Stephenson 1994; MacVean 1983), it is probably preferable to retain hyperdiffusion and to accept the error made by neglecting horizontal dissipation, though it is important to know what the error is.

Section 2 gives a short description of the relevant parts of the ECHAM model. The new and old dissipative heating formulations are briefly recapitulated in the appendixes. In section 3 the effect of exchanging them will be described using ECHAM4/L39 (Land et al. 2002). In section $3 c$, these results will be compared to corresponding estimates obtained from the ECHAM4/ L19 model (Roeckner et al. 1996, 1999). In addition, in section $3 \mathrm{~d}$ we will isolate the separate effects of including horizontal dissipation and changing the parameterization of vertical dissipation.

\section{Model description and significance tests}

The ECHAM4 model (Roeckner et al. 1996, 1999) has been used with 19 vertical levels, ECHAM4/L19, and with 39 vertical levels, ECHAM4/L39DLR (Land et al. 2002). Both model versions have their uppermost full level at $10 \mathrm{hPa}$, with the L39 version having better resolution mainly around the tropopause. ECHAM4, like the newer ECHAM5 (Roeckner et al. 2003), includes a vertical dissipation that assumes a local equivalence of the rate of change of kinetic energy due to vertical momentum diffusion and dissipative heating. The horizontal diffusion is a hyperdiffusion $\left(\nabla^{10}\right)$ up to about $200 \mathrm{hPa}$ above which the sponge layer is imposed. There the order of the diffusion is gradually decreased to $\nabla^{2}$ at the top two model levels in order to avoid spurious wave reflection. Any dissipative heating due to horizontal momentum diffusion is ignored. 
TABLE 1. Experiments.

\begin{tabular}{|c|c|c|c|c|c|c|}
\hline & \multicolumn{2}{|c|}{ Diffusion } & \multicolumn{2}{|c|}{ Dissipation } & \multirow[b]{2}{*}{ L19 } & \multirow[b]{2}{*}{ L39 } \\
\hline & Horizontal & Vertical & Horizontal & Vertical & & \\
\hline Control expt & $\nabla^{2}$ & ECHAM & & ECHAM & $20 \mathrm{yr}$ & $20 \mathrm{yr}$ \\
\hline Horizontal dissipation expt & $\nabla^{2}$ & ЕСНАM & New & ЕСНАM & $20 \mathrm{yr}$ & \\
\hline Horizontal and vertical dissipation expt & $\nabla^{2}$ & ECHAM & New & New & $20 \mathrm{yr}$ & $20 \mathrm{yr}$ \\
\hline
\end{tabular}

For our study, the horizontal diffusion has been changed to an angular-momentum-conserving $\nabla^{2}$ diffusion (Becker 2001; see also appendix A) for both model versions, ECHAM4/L19 and ECHAM4/L39DLR. The diffusion coefficient used for the horizontal diffusion (Fig. 8) increases above $100 \mathrm{hPa}$ strongly, prescribing a sponge layer. Note that the vertical diffusion as included in ECHAM was not changed since its vertical discretization is already consistent with Becker (2003). With this setup, control experiments have been run. Additional runs including horizontal dissipation (appendix A; Becker 2001) and the new vertical dissipation [Eqs. (B1)-(B3) of appendix B; Becker 2003] have been performed with ECHAM4/L19 and with ECHAM4/ L39DLR in order to examine the sensitivities of the models to exchanging both dissipation schemes. In those experiments, the original ECHAM vertical dissipation has been switched off and the new vertical dissipation has been implemented. To isolate the effects of including horizontal dissipation and of changing the representation of vertical dissipation the L19 model has been run with horizontal dissipation included and the original ECHAM vertical dissipation [Eq. (B6) of appendix B] retained. The ECHAM4/L19 and ECHAM4/ L39DLR were both integrated for $20 \mathrm{yr}$. Table 1 summarizes the model configurations of the respective simulations. In sections $3 b$ and $3 c$ the sensitivity of the ECHAM model to changing both dissipation schemes will be analyzed by comparing the results of the horizontal and vertical dissipation experiment with the control experiment. Section $3 \mathrm{~d}$ explores the sensitivity of the model to including horizontal dissipation by comparing the horizontal dissipation experiment with the control experiment.

Significance tests examining the difference fields of two particular model runs have been performed. Differences are assumed to be significantly larger than zero when they exceed a certain threshold associated with a $95 \%$ confidence interval (or $90 \%$ in the case of the change of advective heating) according to the twosided student's $t$ test statistic. The number of independent samples has been calculated, accounting for serial dependence in the time series (Wilks 1995).

\section{Sensitivity of ECHAM to changes in the dissipative heating schemes}

\section{a. Dissipative heating patterns in ECHAM4/L39 (Fig. 1)}

First, the differences between the original ECHAM vertical dissipation formulation, used in the control experiment (see Table 1), and the new vertical dissipation formulation, used in the horizontal and vertical dissipation experiment shall be discussed. To allow for a better comparison of the two vertical dissipation formulations, both heating rates were diagnosed in the horizontal and vertical dissipation experiment, but only the new vertical dissipation was fed into the temperature tendency equation. The dissipative heating patterns due to the original ECHAM parameterization and due to the new parameterization in ECHAM4/L39 are shown in Figs. 1a-d for December-February (DJF) and June-August (JJA). The patterns of the two vertical dissipative heating rates are very similar in the zonal mean. Maximum values are found in the boundary layer and in the tropical and subtropical middle troposphere of the winter hemisphere. In the lowertropospheric midlatitudes vertical dissipation in the $\mathrm{NH}$ is stronger than in the SH. This is likely to be connected with the difference in land coverage of the two hemispheres. The structure of the dissipative heating patterns for JJA (Figs. 1b,d) is roughly a mirror image of the heating pattern in DJF (Figs. 1a,c).

The new vertical dissipation distributes the heating over a larger altitude range than the original ECHAM dissipation when calculating the two dissipation rates from the same input fields. The former is clearly stronger in the Tropics and subtropics at higher altitudes than the original ECHAM dissipation. The latter is concentrated in the boundary layer below $900 \mathrm{hPa}$ exceeding the new vertical dissipation by far. Around 850 $\mathrm{hPa}$ and farther poleward, the new vertical dissipation is slightly weaker than the original ECHAM one in both hemispheres and seasons. The change in the vertical dissipation scheme thus contributes to the differential heating by acting to increase the midlatitude lower-tropospheric temperature gradient. This is espe- 
(a)

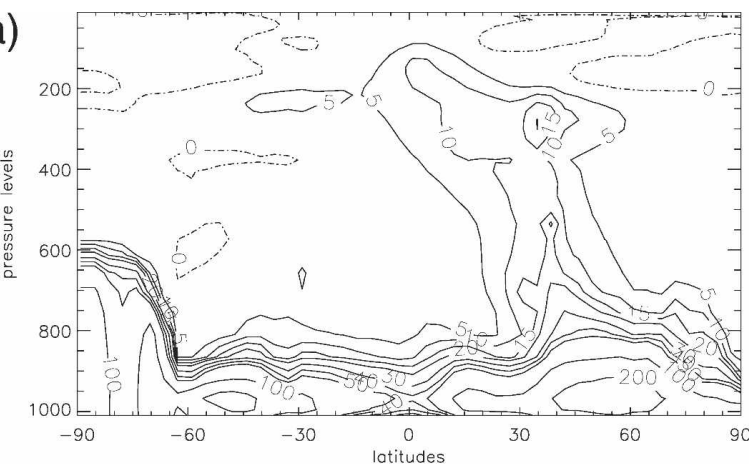

(c)

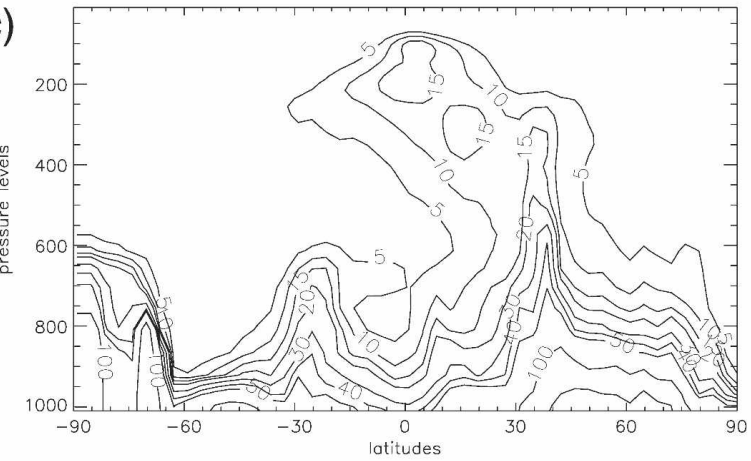

(e)

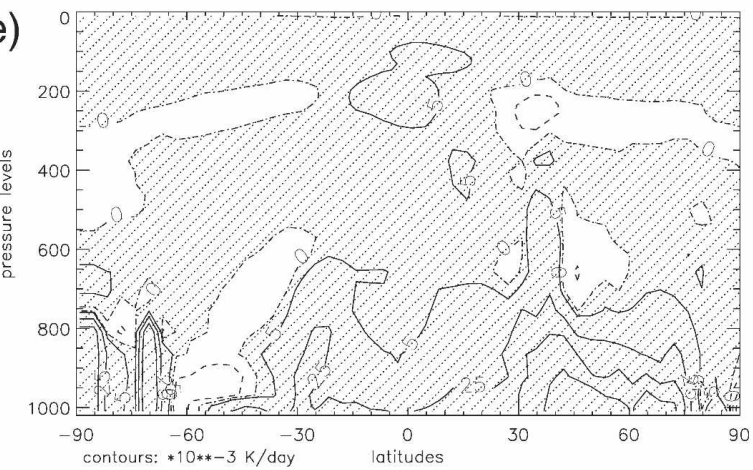

(g)

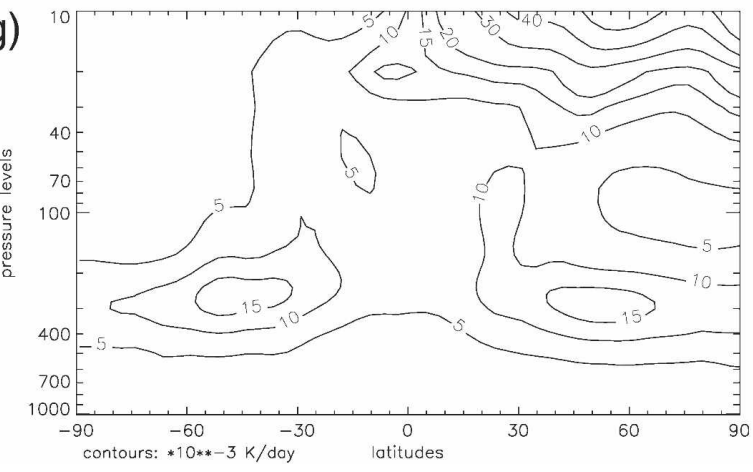

(b)

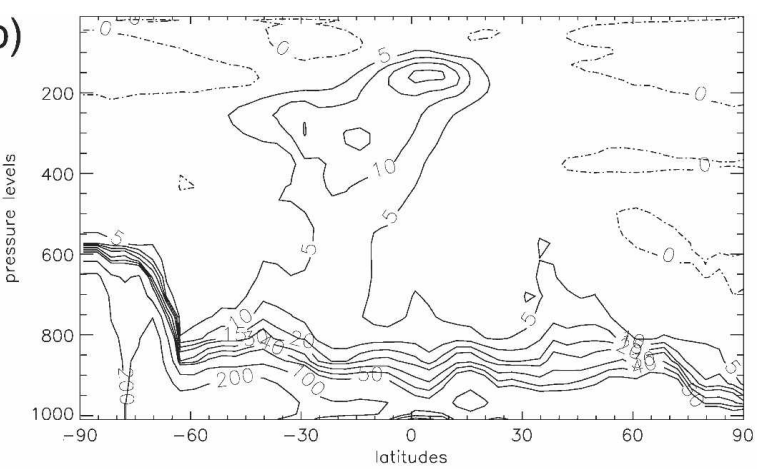

(d)

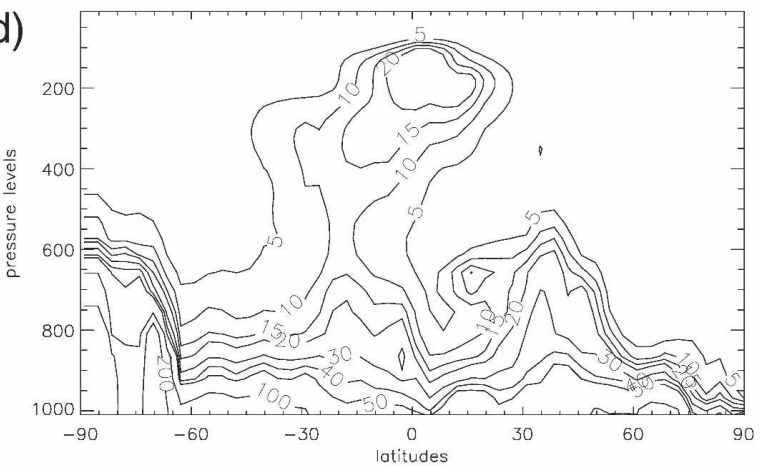

(f)

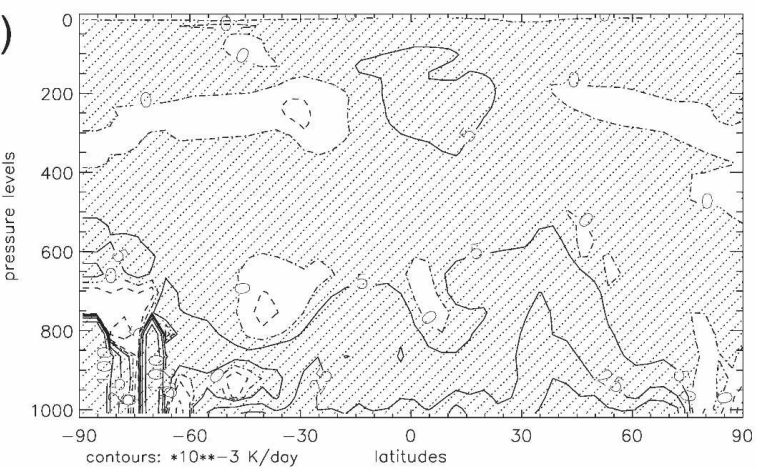

(h)

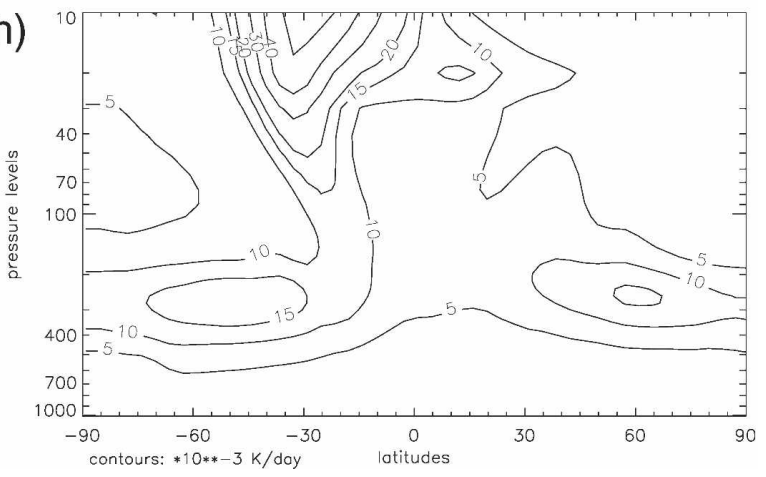

FIG. 1. Dissipative heating in ECHAM4/L39 due to vertical diffusion determined by (a), (b) the original ECHAM parameterization and (c), (d) the new parameterization in the horizontal and vertical dissipation experiment. (e), (f) The difference of the new scheme, as analyzed from the horizontal and vertical dissipation experiment minus original ECHAM scheme, as diagnosed from the control experiment. (g), (h) Dissipation due to horizontal diffusion for (left) DJF and (right) JJA. Contours are 5, 10, 15, 20, 30, 40, 50, 100, $200 \times 10^{-3} \mathrm{~K} \mathrm{day}^{-1}$, except in (e) and (f) where contours are $-100,-50,-25,-5,0,5,25,50,100 \times 10^{-3} \mathrm{~K}_{\text {day }}{ }^{-1}$. Shaded areas in (e) and (f) indicate areas in which dissipation due to the new parameterization is larger than that due to the original ECHAM one. 
cially the case for the Northern Hemisphere. Another difference is that for the original ECHAM parameterization negative heating values can frequently be found whereas the new parameterization is positive definite. Local differences between vertical dissipation as computed by the original ECHAM parameterization and the new parameterization are stronger in the northern than in the southern midlatitudes (not shown), but have a tendency to average out in the zonal mean.

When comparing vertical dissipation due to the original ECHAM scheme as analyzed in the control experiment with the vertical dissipation of the horizontal and vertical dissipation experiment, a quite different response of the vertical dissipation field is obtained (Figs. $1 \mathrm{e}, \mathrm{f})$. Vertical dissipation is larger due to the new parameterization nearly everywhere except in the area of the southern midlatitudes. The maximum increase of dissipative heating can be found at the surface, with the largest changes in the northern storm-track area in both seasons. Above $800 \mathrm{hPa}$, the change of dissipative heating due to the two parameterizations is quite close to the difference in the heating patterns shown in Figs. $1 \mathrm{a}, \mathrm{c}$ and $1 \mathrm{~b}, \mathrm{~d}$. Therefore, our results compare well with the results of Boville and Bretherton (2003, their Fig. 3 for the annual mean response) who also find an increased vertical dissipation at the surface using the new scheme, though their maximum increase is located in the southern storm-track area. Apparently, employing the new vertical dissipation parameterization rather than using the original ECHAM dissipation causes an increase in boundary layer turbulence that is parameterized by vertical diffusion. This may be because the original ECHAM dissipation heats mainly the area close to the surface suppressing surface heating.

Horizontal dissipation (Figs. $1 \mathrm{~g}, \mathrm{~h}$ ) is at a maximum in the extratropical midlatitudes, in the area of the storm tracks, and in the sponge layer of the winter hemisphere. During the respective summer and winter seasons, it is stronger in the southern than in the northern upper troposphere. This is connected with the storm tracks being stronger in the southern than in the Northern Hemisphere for both seasons (Land et al. 2002). In the sponge layer, horizontal dissipation is spread out over a larger range of latitudes in the northern winter hemisphere than in the southern winter hemisphere due to the larger variability of the northern polar vortex.

\section{b. Sensitivity of ECHAM4/L39 (Fig. 2)}

Figure 2 shows the temperature response in the L39 model due to changing both dissipation schemes, that is, the difference between the climate of the horizontal and vertical dissipation experiment and the control experiment for DJF and JJA. The temperature response (a)

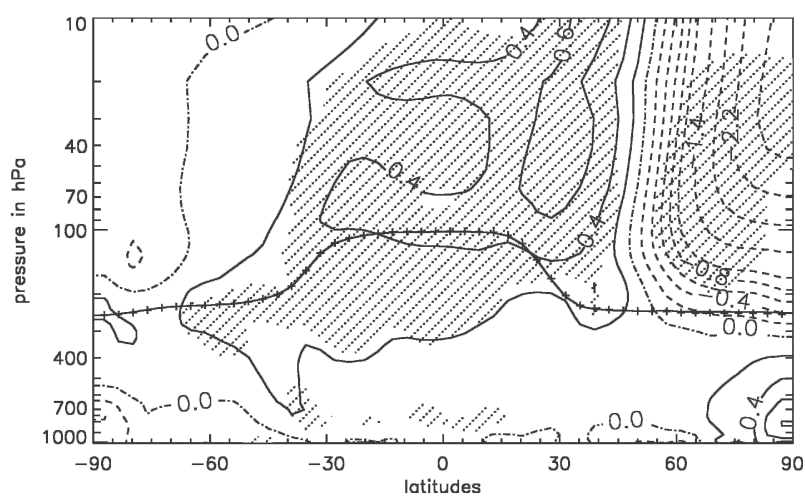

(b)

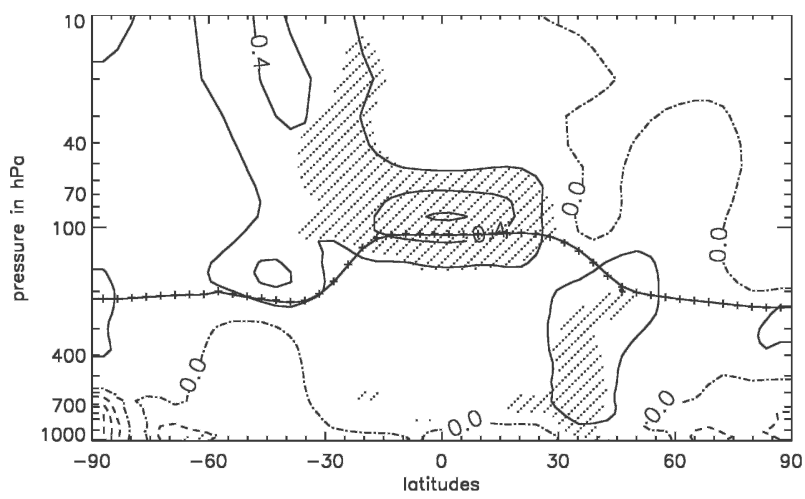

FIG. 2. Temperature response due to exchanging the dissipation schemes in ECHAM4/L39 (horizontal and vertical dissipation experiment minus control experiment) for (a) DJF and (b) JJA. The height of the tropopause is indicated by a solid line. Isolines are drawn at $0.2 \mathrm{~K}$ intervals up to $1 \mathrm{~K}$ and then every $0.4 \mathrm{~K}$. Shaded areas indicate changes that are significant at the $95 \%$ confidence level.

is negative in the northern winter polar stratosphere (Fig. 2a), implying a strengthening of the vortex. The cooling of the polar vortex well exceeds $1 \mathrm{~K}$. This sensitivity is only partly significant because of the strong internal variability and serial correlation in the temperature time series in this area. Additionally, the model simulates a statistically significant warming of about $0.5 \mathrm{~K}$ along the tropopause in the Tropics and in the southern midlatitudes during DJF. This warming is too weak to change the height of the tropopause significantly. Connected with it is a general moistening of the stratosphere by about 5\% (not shown), consistent with the warming of the tropical cold point. The moistening is most pronounced in the area of the cold point.

In Southern Hemispheric winter (JJA), the new dissipation schemes lead to a slight but significant warming around the tropical tropopause of about $0.5 \mathrm{~K}$ (Fig. $2 \mathrm{~b})$. Again, this warming is connected with a general moistening of the stratosphere of about $5 \%$, consistent with the warming of the cold point (Fig. 3). 
(a)

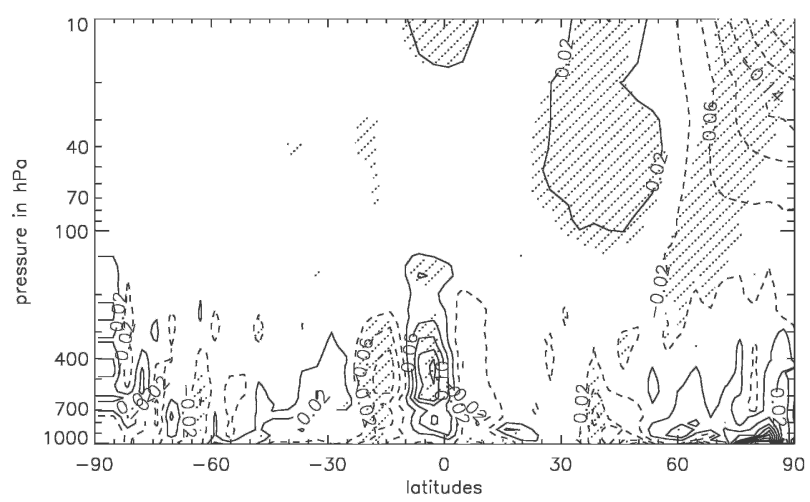

(b)

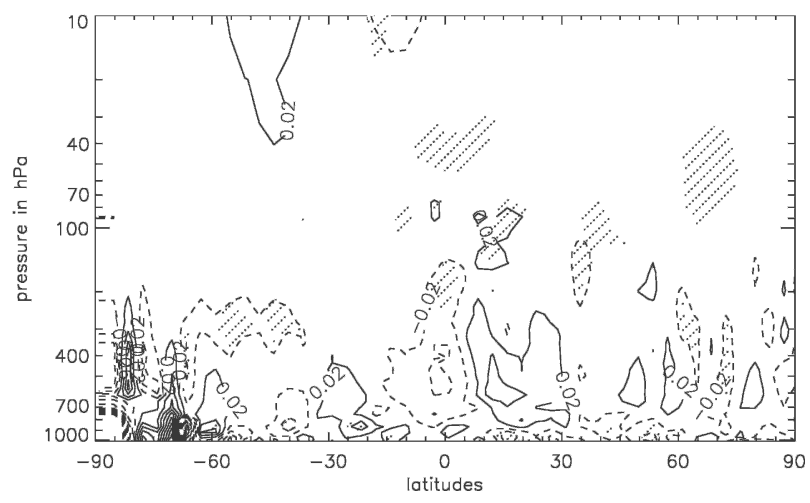

FIG. 3. Response of dynamic heating due to exchanging the dissipation schemes (horizontal and vertical dissipation experiment minus control experiment) in ECHAM4/L39 for (a) DJF and (b) JJA. Contour interval is $0.04 \mathrm{~K} \mathrm{day}^{-1}$. Shaded areas indicate changes that are significant at the $90 \%$ significance level.

The hemispheric asymmetry of the temperature response of the winter polar stratosphere can be traced back to an asymmetry in the dynamic heating (advection plus adiabatic heating). In the extratropical upper troposphere and stratosphere, dynamic heating corresponds to the strength of the residual circulation (Andrews et al. 1987) and thus reflects the quasigeostrophic wave drag (Holton et al. 1995). Figure 3a indicates that the cooling of the northern winter polar vortex is dominated by a weakening of the residual circulation. In southern winter, dynamic heating is not changed when implementing the new dissipation schemes, and consequently the temperature response is close to zero.

In section $3 \mathrm{~d}$ we show that in northern winter the change in the residual circulation, which is driven by breaking Rossby waves forced in the troposphere, is an indirect effect of the reformulated vertical dissipation. The differences in the dynamic heating in the polar stratosphere must therefore be due to the tropospheric differences in the vertical dissipation. As in ECHAM4, in the simple GCM of Becker (2003) the wintertime vertical dissipative heating maxima [calculated according to Eq. (B6)] in the lower troposphere are located in the midlatitudinal regions of high sea surface temperatures at the beginning of the storm tracks. Vertical dissipation thus contributes to the forcing of planetary Rossby waves and promotes the growth of baroclinic waves, which again feed back on the large-scale waves. Exchanging the vertical dissipation scheme results in a reduction of the heating in the areas of high sea surface temperatures and therefore in a more zonally symmetric heating in the boundary layer in northern winter. This acts to decrease the land-sea heating contrast and with it the Rossby wave forcing and the residual circulation. In southern winter, dissipative heating is close to zonally symmetric, and local differences between the two dissipation schemes are much smaller so that the Rossby waves are not changed. As a result, dynamic heating in the southern stratosphere is hardly influenced by changes in the dissipation scheme (see Fig. 3b).

The radiative imbalance at the top of the atmosphere is slightly reduced when changing the dissipative heating parameterization. Comparing the horizontal and vertical dissipation experiment with the control experiment, this reduction amounted to $0.35 \mathrm{~W} \mathrm{~m}^{-2}$.

\section{c. Sensitivity of ECHAM4/L19}

The dissipative heating patterns in the ECHAM4/ L19 are very similar to those in the ECHAM4/L39 for both winter seasons. Both the new and the original ECHAM vertical dissipation are generally weaker in the L19 model version (Fig. 4a for the new vertical dissipation in DJF) than in the L39 model version (Fig. 1c). Nevertheless, the differences between the original ECHAM formulation and the new formulation are again very similar to those seen in the L39 model; that is, the new parameterization distributes the heating more evenly over the lower troposphere and increases slightly the temperature gradient in the lower troposphere. The differences between the two schemes are smaller than in the L39 model. Horizontal dissipation in the upper troposphere is stronger in the L19 model (Fig. 4b for DJF) than in the L39 model (Fig. 1g) by $10 \%-20 \%$, while in the sponge layer it is somewhat weaker for both $\mathrm{NH}$ and $\mathrm{SH}$ winter due to less wave propagation into the stratosphere.

The JJA temperature response to changing both dissipation schemes in the L19 model (Fig. 5b) is similar to the corresponding response in the L39 model (Fig. 2b). The area around the tropopause is slightly but significantly warmed. Maximum warming occurs in the subtropical tropopause area.

The temperature signal for northern winter, DJF 
(a)

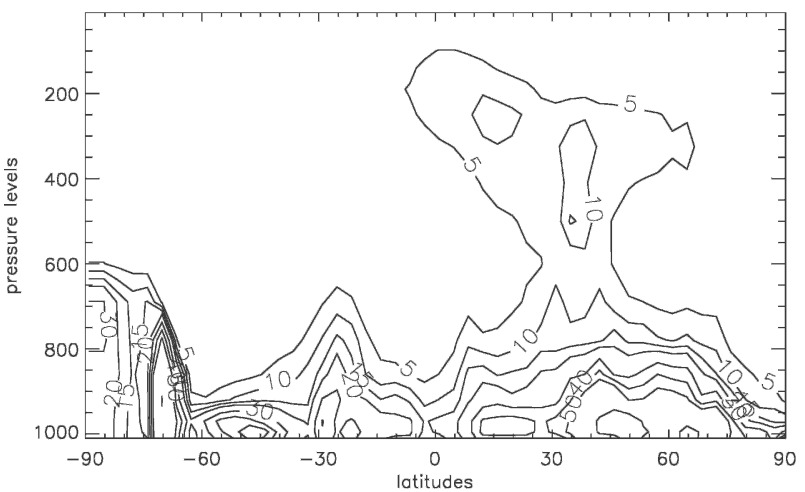

(b)

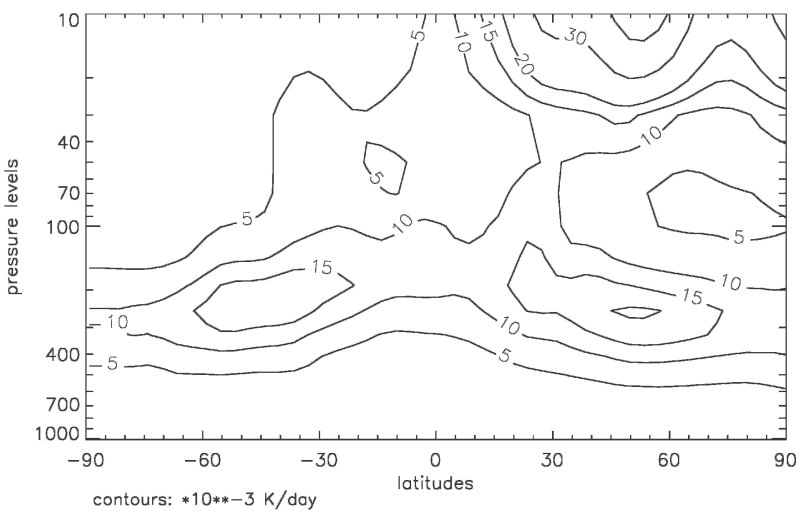

FIG. 4. Dissipative heating in ECHAM4/L19 due to (a) vertical momentum diffusion determined by the new parameterization and due to (b) horizontal momentum diffusion for DJF. Contours are $5,10,15,20,30,40,50,100,200 \times 10^{-3} \mathrm{~K} \mathrm{day}^{-1}$.

(Fig. 5a), on the other hand, is different from the L39 model result (Fig. 2a). As in the L39 model version, there is a significant warming in the area around the tropopause in the Tropics and subtropics. The temperature response in the winter polar vortex, on the other hand, is very different from that of the L39 model version. In the L19 model version, the temperature in the northern polar vortex does not change significantly and dynamic heating in this area (not shown) is only slightly negative. Therefore, direct heating due to horizontal dissipation and cooling due to a slight change in dynamic heating cancel out in the northern winter vortex area in the L19 model version.

\section{d. Isolating the effects of horizontal and vertical dissipation}

In the following, the effects of horizontal and vertical dissipation shall be isolated using the ECHAM4/L19 model. For this purpose the horizontal dissipation experiment, in which horizontal dissipation was included (a)

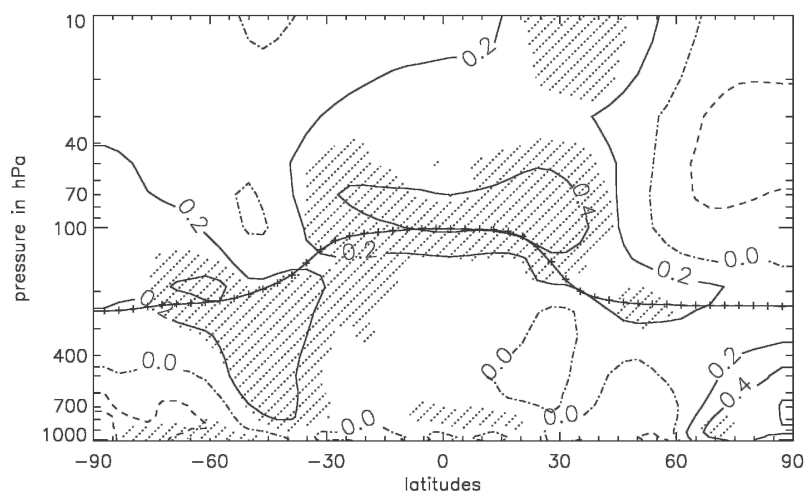

(b)

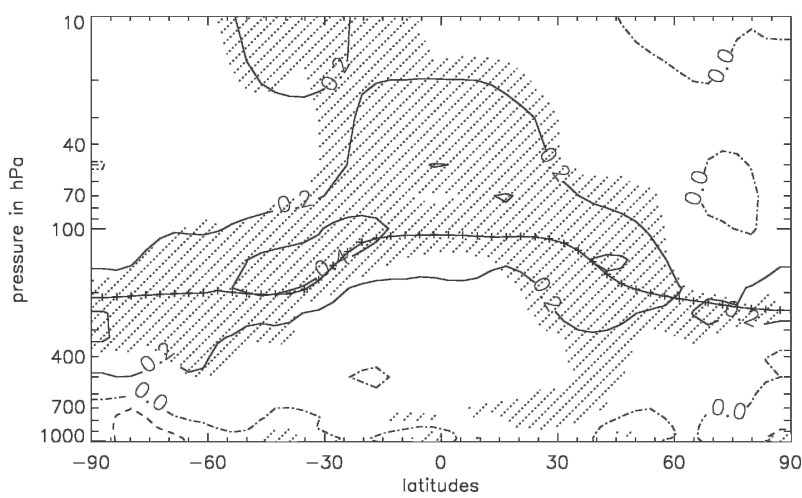

FIG. 5. As in Fig. 2, but for ECHAM4/L19. Contour interval is $0.2 \mathrm{~K}$.

but the original ECHAM vertical dissipation was retained (see Table 1), was compared with the control experiment. Likewise, the effect of changing the vertical dissipation parameterization is isolated by comparing the horizontal and vertical dissipation experiment with the horizontal dissipation experiment.

The DJF temperature response to the inclusion of horizontal dissipation is shown in Fig. 6a. In northern winter the area along the tropopause is warmed. The temperature also rises in the northern polar vortex, exceeding $1 \mathrm{~K}$ in the top few levels. This warming is caused directly by horizontal dissipation, as well as by increased dynamic heating (not shown) associated with somewhat enhanced planetary wave activity. The latter is also indicated by the warming at high and polar winter latitudes in the lower troposphere (Fig. 7).

The DJF temperature difference resulting from the change in vertical dissipation (Fig. 7a) is a cooling in the Northern Hemispheric extratropics, which is partly significant in the lower stratosphere. This response is caused by anomalous dynamic cooling in the northern high latitudes (Fig. 7b), indicating a weakened residual circulation. This means that the effect of the horizontal 
(a)

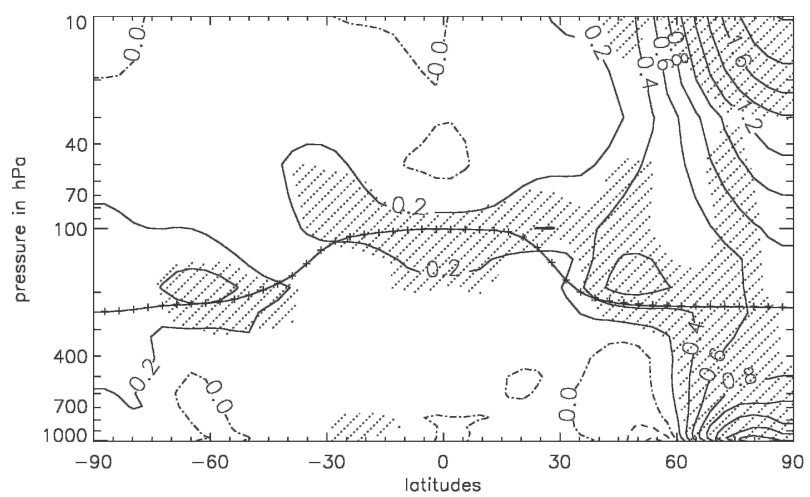

(b)

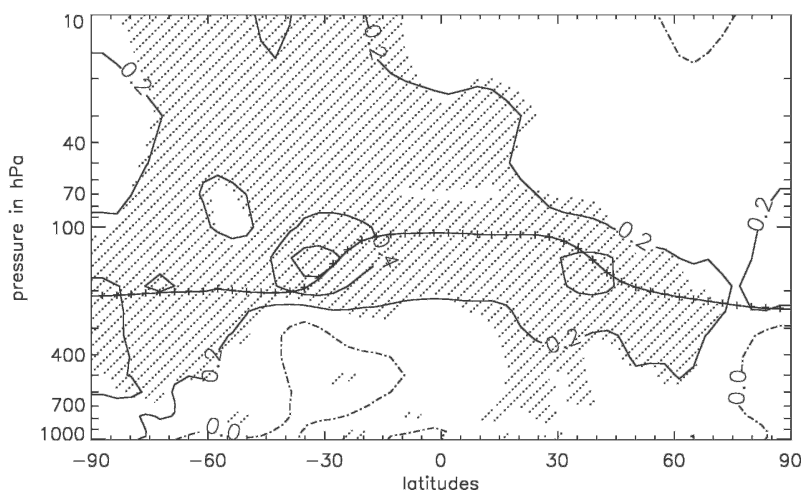

FIG. 6. As in Fig. 5 but response to including horizontal dissipation.

dissipation, to warm both the northern winter stratosphere and the high latitudes in the troposphere (Fig. $6 a)$, is counterbalanced by the cooling associated with exchanging the original ECHAM vertical dissipation for the new one (Fig. 7a). As a result, the temperature is not significantly changed due to the combination of both new dissipation schemes (Fig. 5a).

In southern winter (JJA), the area around the tropopause is slightly but significantly warmed due to the inclusion of horizontal dissipation (Fig. 6b). Maximum values of around $0.5 \mathrm{~K}$ are attained in the area of the subtropical tropopause. Comparing Fig. 6b with Fig. 5b, it can be seen that the effect of exchanging the vertical dissipation parameterization is negligible; that is, the details of the vertical dissipation do not significantly affect the temperature field during southern winter.

The slight warming around the tropopause seems to be a robust consequence of the imposed changes in the dissipation schemes. In southern winter, when the change in the vertical dissipation does not change the temperature field, both model versions display a similar response to changing both the vertical and horizontal dissipation scheme. The warming depends on the (a)

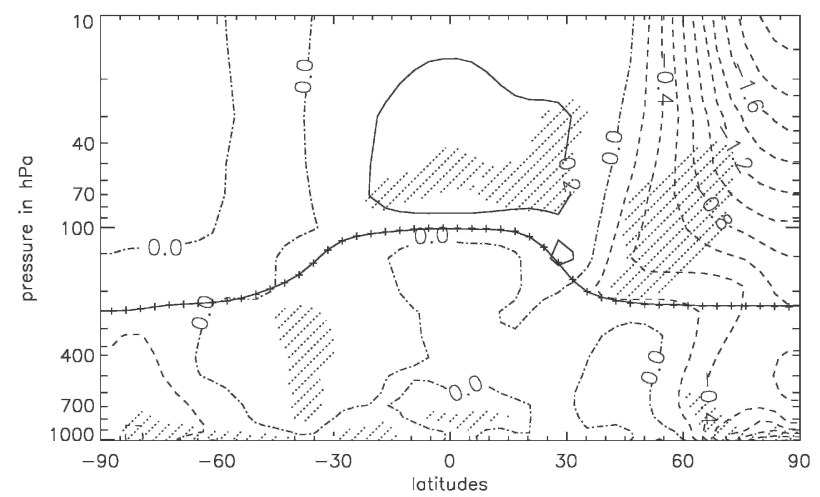

(b)

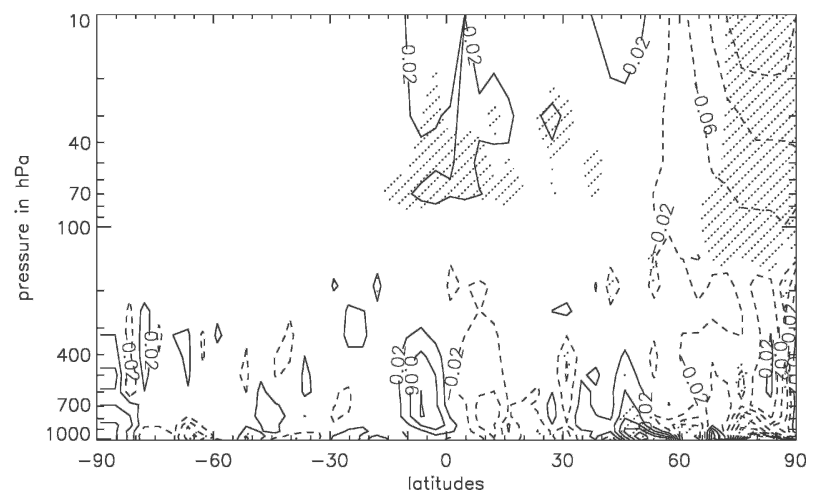

FIG. 7. (a) Temperature response (DJF) to exchanging the vertical dissipation and (b) the associated response in dynamic heating. Contour interval is $0.2 \mathrm{~K}$ in (a) and $0.04 \mathrm{~K}$ day $^{-1}$ in (b).

strength of the horizontal dissipation, which is weaker in the L39 model version than in the L19 model. Accordingly, the heating along the extratropical tropopause is weaker in the L39 model version. The response of the northern winter polar vortex is, however, sensitive to the details of the vertical dissipation in the lower troposphere, which have a remote effect on the residual circulation in the stratosphere. The weakening of the residual circulation is much more pronounced in the L39 model version.

\section{Conclusions}

The paper's aim is to examine whether a reformulation of dissipative (frictional) heating, in order to achieve consistency with basic hydrodynamic constraints, can affect the climate simulated by a sophisticated climate model, such as ECHAM4. To study the model's sensitivity, the horizontal diffusion had to be changed to a $\nabla^{2}$ diffusion. Dissipative heating due to horizontal momentum diffusion (horizontal dissipation), which is usually ignored in climate models, has 
been introduced in ECHAM4. Likewise, the dissipative heating due to vertical momentum diffusion (vertical dissipation) has been reformulated.

Horizontal dissipation is at a maximum in the area of the storm tracks $\left(0.02 \mathrm{~K} \mathrm{day}^{-1}\right)$ and in the sponge layer of the winter hemisphere. Including this term in ECHAM4 leads primarily to a slight but statistically significant warming of the area around the tropopause (up to about $0.4 \mathrm{~K}$ ). Furthermore, the northern winter polar vortex is warmed by more than $1 \mathrm{~K}$. This warming is in part caused directly by horizontal dissipation, and in part by enhanced dynamic heating (advection plus adiabatic heating).

The pattern of the new vertical dissipation is similar to the one usually included in ECHAM. The new vertical dissipation scheme distributes the dissipative heating more evenly over the troposphere than the original ECHAM vertical dissipation when diagnosed in the same model run. In particular, the new parameterization does not display any negative dissipation rates, as the original ECHAM parameterization does even in the climatological zonal mean. When analyzing vertical dissipation from the runs where the respective schemes were used, vertical dissipation due to the new scheme is nearly everywhere larger than the original ECHAM dissipation. Maximum changes are found at the surface in the area of the Northern Hemispheric storm track. Using the new vertical dissipation increases the amount of kinetic energy transformed into inner energy by the vertical diffusion/dissipation scheme. This result mostly agrees with the results of Boville and Bretherton (2003) who found an increased vertical dissipation as well though their maximum increase was located in the Southern Hemispheric storm-track area. Our reformulation of the vertical dissipation cannot heat the stratosphere directly since vertical diffusion in ECHAM is relevant only in the lower troposphere and in the tropical and subtropical winter middle troposphere. Nevertheless, it can affect the stratosphere indirectly by altering the Rossby wave forcing and thereby the Rossby wave drag, which drives the residual circulation.

Exchanging the original ECHAM vertical dissipation for the new formulation leads to a weakening of the residual circulation in Northern Hemispheric winter and therefore to a cooling and strengthening of the polar vortex. This remote effect is much more pronounced in the L39 model version. This cooling in the northern polar vortex partly cancels out (L19) or even reverses (L39) the Northern Hemispheric warming due to the inclusion of horizontal dissipation. In southern winter, the change of the vertical dissipation scheme does not have a significant effect on the temperature field, neither directly in the troposphere nor remotely in the stratosphere. Accordingly, the L19 and L39 model versions show quite similar responses to changing both dissipation schemes during southern winter.

The imposed changes of the dissipation scheme affect the simulated climate significantly. The need to choose a $\nabla^{2}$-horizontal diffusion, in order for horizontal dissipation to be positive definite, reduces the scale selectivity of the horizontal diffusion scheme and thereby prevents this model modification from being routinely implemented in a low-resolution climate model. Current work is therefore concentrating on the implementation of a nonlinear $\nabla^{2}$ diffusion and the associated dissipative heating.

Several other energy sinks and sources exist in GCMs-such as diffusive numerics, subgrid processes like convection, neglecting of heating due to phase transitions, and others - that require treatment in order to energetically close a GCM. Recently, attempts to account for those energy losses or to backscatter kinetic energy partly onto the resolved scale have been made in GCMs (Boville and Bretherton 2003; Shutts 2004) but still more attention is needed in many climate models to parameterize those processes consistently.

Acknowledgments. We thank Uli Schlese (MPI Hamburg) for technical help with the ECHAM model and Michael Ponater and Robert Sausen for discussions and support. The study was funded by the BMBF in the DEKLIM program (Cobi Project, 01LD0045).

\section{APPENDIX A}

\section{Horizontal Dissipative Heating}

According to the symmetric stress tensor formulation of horizontal momentum diffusion given in Becker (2001), the dissipation is only positive definite if the momentum diffusion is of second order. If the diffusion coefficient, $K_{h}$, is specified as a function of height, the tendencies of horizontal momentum and temperature owing to horizontal momentum diffusion may be written as

$$
\begin{aligned}
\left(\partial_{t} \mathbf{v}\right)_{h}= & K_{h}\left(\nabla^{2} \mathbf{v}+\nabla D+2 \mathbf{v} / a^{2}\right), \\
c_{p}^{-1} \epsilon_{h}= & c_{p}^{-1} K_{h}\left[2\left(D-\partial_{\phi} v / a\right)^{2}+2\left(\partial_{\phi} v / a\right)^{2}\right. \\
& \left.+\left(\xi+2 \partial_{\phi} u / a\right)^{2}\right] .
\end{aligned}
$$

Here, $\nabla$ and $\mathbf{v}$ denote the horizontal gradient operator and velocity field, $u$ and $v$ denote the eastward and northward velocity components, $D$ and $\xi$ are horizontal divergence and relative vorticity, $a$ is the earth radius, $c_{p}$ is heat capacity, and $\phi$ is latitude. Equation (A1) 
gives rise to the following tendencies of relative vorticity and horizontal divergence in spectral space:

$$
\begin{gathered}
\left(\partial_{t} \xi_{n m}\right)_{h}=-\frac{K_{h}}{a^{2}}[n(n+1)-2] \xi_{n m}, \\
\left(\partial_{t} D_{n m}\right)_{h}=-\frac{K_{h}}{a^{2}}[2 n(n+1)-2] D_{n m} .
\end{gathered}
$$

In our present version of ECHAM4, the tendencies (A3) and (A4) are, like the horizontal diffusion tendencies in the original model, implemented in spectral space. Furthermore, horizontal diffusion of temperature has been changed to the harmonic scheme using

$$
\left(\partial_{t} T_{n m}\right)_{h}=-\frac{K_{h}}{2 a^{2}} n(n+1) T_{n m} .
$$

The horizontal dissipation (A2) is computed in grid space and added to the dynamic temperature tendencies. The required horizontal derivatives of $u$ and $v$ are computed using the spectral transform method. Note that (A2)-(A5) are applied at the model's hybrid surfaces, that is, $K_{h}=K_{h}(\eta)$. Figure A1 shows the assumed vertical profile of $K_{h}$, which approaches zero in the lower troposphere in order to avoid coordinate transformation errors in regions of steep orography (Becker 2003). The zonal mean climatology using second-order diffusion and the diffusion coefficient shown in Fig. A1 is very close to the standard ECHAM4 wind field (Roeckner et al. 1996) with slightly higher wind speeds in the northern winter sponge layer (Fig. A1).

\section{APPENDIX B}

\section{Vertical Dissipative Heating}

The discretized vertical momentum diffusion tendencies are written as in Becker [2003, Eq. (15)]:

$$
\begin{aligned}
& \mathbf{Z}_{l}= \\
& \frac{-g}{\Delta p_{l}} \begin{cases}\left(\rho K_{z} \partial_{z} \mathbf{v}\right)_{l+1 / 2} & l=1 \\
\left(\rho K_{z} \partial_{z} \mathbf{v}\right)_{l+1 / 2}-\left(\rho K_{z} \partial_{z} \mathbf{v}\right)_{l-1 / 2} & l=2 \ldots N-1 . \\
C \rho_{l} \mathbf{v}_{l}-\left(\rho K_{z} \partial_{z} \mathbf{v}\right)_{l-1 / 2} & l=N\end{cases}
\end{aligned}
$$

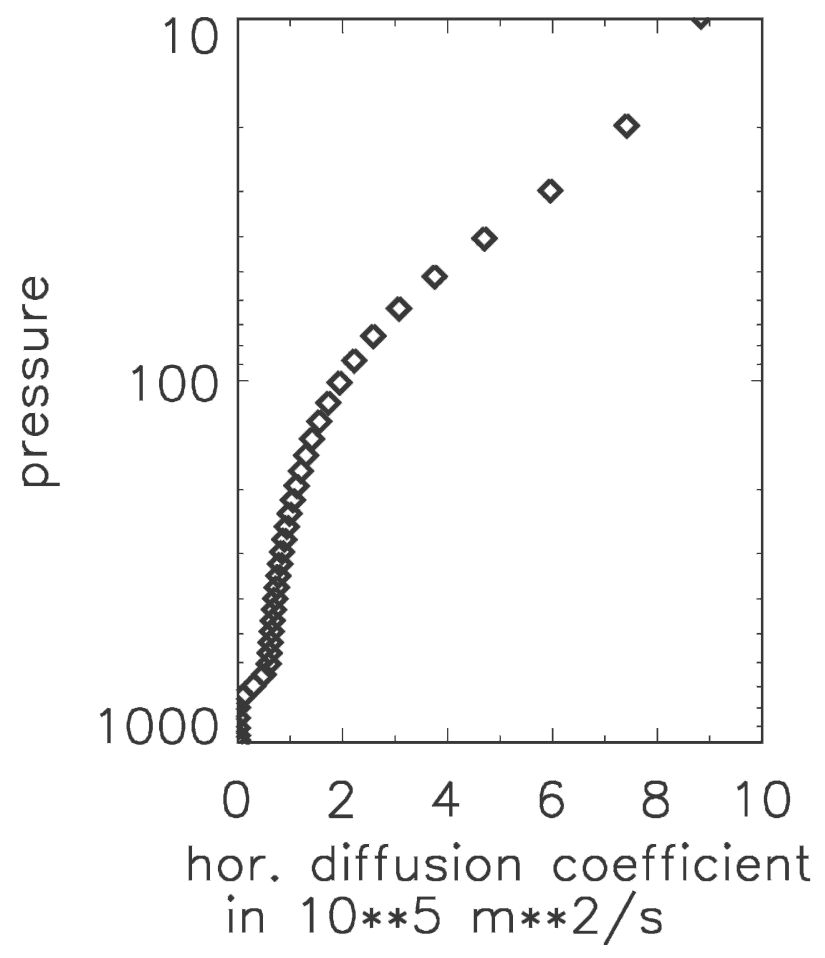

FIG. A1. Horizontal diffusion coefficient $\left(10^{5} \mathrm{~m}^{2} \mathrm{~s}^{-1}\right)$.

Here, the index $l$ denotes full hybrid levels such that $l+1 / 2$ and $l-1 / 2$ denote the intermediate half levels. The total number of full hybrid levels is $N$. The pressure differences are $\Delta p_{l}=p_{l+1 / 2}-p_{l-1 / 2}$ with $p_{1 / 2}=0$ and $p_{\mathrm{N}+1 / 2}=p_{s}$, where $p_{s}$ is surface pressure. The density at full levels, $\rho_{l}$, is calculated from the equation of state; $K_{z}$ is the vertical diffusion coefficient, and $C=$ $C_{D}|\mathbf{v}|$ is the surface drag coefficient. Furthermore we have used

$$
\left(\rho K_{z} \partial_{z} \mathbf{v}\right)_{l+1 / 2}=\rho_{l+1 / 2}\left(K_{z}\right)_{l+1 / 2}\left(\partial_{z} \mathbf{v}\right)_{l+1 / 2}
$$

for $l=2, \ldots, N-1$.

An energy-conserving vertical discretization of the hydrodynamic vertical dissipation-more exactly, of the transfer of mean kinetic energy into turbulent kinetic energy-is given by

$$
\begin{aligned}
& \epsilon_{z l}= \\
& \quad \frac{1}{2 \Delta p_{l}} \begin{cases}\left(\rho K_{z} \partial_{z} \mathbf{v}\right)_{l+1 / 2} \cdot\left(\partial_{z} \mathbf{v}\right)_{l+1 / 2}\left(p_{l+1}-p_{l}\right) / \rho_{l+1 / 2} & l=1 \\
\left(\rho K_{z} \partial_{z} \mathbf{v}\right)_{l-1 / 2} \cdot\left(\partial_{z} \mathbf{v}\right)_{l-1 / 2}\left(p_{l}-p_{l-1}\right) / \rho_{l-1 / 2}+\left(\rho K_{z} \partial_{z} \mathbf{v}\right)_{l+1 / 2} \cdot\left(\partial_{z} \mathbf{v}\right)_{l+1 / 2}\left(p_{l+1}-p_{l}\right) / \rho_{l+1 / 2} & l=2 \ldots N-1 \\
\left(\rho K_{z} \partial_{z} \mathbf{v}\right)_{l-1 / 2} \cdot\left(\partial_{z} \mathbf{v}\right)_{l-1 / 2}\left(p_{l}-p_{l-1}\right) / \rho_{l-1 / 2}+2 g C \rho_{l} \mathbf{v}_{l}^{2} & l=N\end{cases}
\end{aligned}
$$


using

$$
\begin{aligned}
\left(\partial_{z} \mathbf{v}\right)_{l+1 / 2} & =-g \rho_{l+1 / 2}\left(\mathbf{v}_{l+1}-\mathbf{v}_{l}\right)\left(p_{l+1}-p_{l}\right)^{-1}, \\
\rho_{l+1 / 2} & =\left(\rho_{l+1}+\rho_{l}\right) / 2,
\end{aligned}
$$

for $l=2, \ldots, N-1$. This formulation is taken from Becker [2003, Eq. (16)] and is equivalent to that proposed by Boville and Bretherton [2003, Eqs. (43)-(45)]. The energy conversion rate (B3) divided by $c_{p}$ is added to the vertical diffusion tendency of temperature. Note that there is a slight difference in the implementation of vertical dissipation here and in Boville and Bretherton (2003). Whereas here dissipative heating was calculated from the variables at one time step, Boville and Bretherton (2003) include the time development of the wind in their formulation, therefore conserving energy exactly while sacrificing positive definiteness of the dissipation.

In the original ECHAM4 vertical momentum diffusion is discretized as in (B1) but dissipation due to vertical momentum diffusion, as in many other climate models, corresponds to

$$
\epsilon_{z l-\text { original }}=-\mathbf{v}_{l} \cdot \mathbf{Z}_{l} .
$$

This conventional formulation conserves energy, yet it can heat or cool.

The vertical diffusion coefficient in ECHAM is computed by solving a tendency equation for the turbulent kinetic energy, and the surface drag coefficient is computed in a diagnostic way (Brinkop and Roeckner 1995). In the future we plan to take the dynamic character of this turbulence model fully into account. This requires us to include the tendency of the turbulent kinetic energy in the thermodynamic equation of motion, as well as to replace the shear production by (B3).

\section{REFERENCES}

Andrews, D. G., J. R. Holton, and C. B. Leovy, 1987: Middle Atmosphere Dynamics. Academic Press, 489 pp.

Becker, E., 2001: Symmetric stress tensor formulation of horizontal momentum diffusion in global models of atmospheric circulation. J. Atmos. Sci., 58, 269-282.

_ 2003: Frictional heating in global climate models. Mon. Wea. Rev., 131, 508-520.

Boville, B. A., and C. S. Bretherton, 2003: Heating and kinetic energy dissipation in the NCAR Community Atmosphere Model. J. Climate, 16, 3877-3887.
Brinkop, S., and E. Roeckner, 1995: Sensitivity of a general circulation model to parameterizations of cloud-turbulence interactions in the atmospheric boundary layer. Tellus, 47A, 197-220.

DKRZ, 1992: The ECHAM3 atmospheric general circulation model. Tech. Rep. 6, Deutsches Klimarechenzentrum, Hamburg, Germany, 187 pp.

Hamilton, K., 1996: Comprehensive meteorological modeling of the middle atmosphere: A tutorial review. J. Atmos. Terr. Phys., 58, 1591-1627.

Holton, J. R., P. H. Haynes, M. E. McIntyre, A. R. Douglass, R. B. Rood, and L. Pfister, 1995: Stratosphere-troposphere exchange. Rev. Geophys., 33, 403-439.

Kiehl, J. T., J. J. Hack, G. B. Bonan, B. A. Boville, B. P. Briegleb, D. L. Williamson, and P. J. Rasch, 1996. Description of the NCAR community climate model (COM3). NCAR Tech. Note NCAR/TN-420+STR, $152 \mathrm{pp}$

Land, C., J. Feichter, and R. Sausen, 2002: Impact of the vertical resolution on the transport of passive tracers in the ECHAM4 model. Tellus, 54B, 344-360.

MacVean, M. K., 1983: The effects of horizontal diffusion on baroclinic development in a spectral model. Quart. J. Roy. Meteor. Soc., 109, 771-783.

Medvedev, A. S., and G. P. Klaasen, 2003: Thermal effects of saturating gravity waves in the atmosphere. J. Geophys. Res., 108, 4040, doi:10.1029/2002JD002504.

Oort, A. H., 1964: On estimates of the atmospheric energy cycle. Mon. Wea. Rev., 92, 483-493.

Pedlosky, J., 1987: Geophysical Fluid Dynamics. Springer-Verlag, $710 \mathrm{pp}$

Roeckner, E., and Coauthors, 1996: The atmospheric general circulation model ECHAM-4: Model description and simulation of present-day climate. MPI Rep. 218, 90 pp.

_ L L. Bengtsson, J. Feichter, J. Lelieveld, and H. Rodhe, 1999: Transient climate change simulations with a coupled atmosphere-ocean GCM including the tropospheric sulfur cycle. $J$. Climate, 12, 3004-3032.

— , and Coauthors, 2003: The atmospheric general circulation model ECHAM5. Part 1: Model description. MPI Rep. 349, $127 \mathrm{pp}$.

Shutts, G., 2004: A stochastic kinetic energy backscatter algorithm for use in ensemble prediction systems. ECMWF Tech. Memo. 449, 26 pp.

Smagorinsky, J., 1993: Some historical remarks on the use of nonlinear viscosities. Large Eddy Simulation of Complex Engineering and Geophysical Flows, B. Galperin and S. A. Orszag, Eds., Cambridge University Press, 3-36.

Stephenson, D. B., 1994: The impact of changing the horizontal diffusion scheme on the northern winter climatology of a general circulation model. Quart. J. Roy. Meteor. Soc., 120, 211-226.

Wilks, D. S., 1995: Statistical Methods in the Atmospheric Sciences. Academic Press, 464 pp. 